Eval uat i on of abrupt energy transfer anrong t urbul ent pl asma st ruct ures usi ng si ngul ar val ue decompositi on

\begin{tabular}{|l|l|}
\hline $\begin{array}{l}\text { j our nal or } \\
\text { publ i cat i on } \mathrm{t} \text { i t l e }\end{array}$ & $\mathrm{Pl}$ asma Physi cs and Cont rol I ed Fusi on \\
\hline vol une & 63 \\
\hline number & 2 \\
\hline page r ange & 025004 \\
\hline year & $2020-12-08$ \\
\hline URL & ht t p: //hdl . handl e. net /10655/00012803 \\
\hline
\end{tabular}




\title{
Evaluation of abrupt energy transfer among turbulent plasma structures using singular value decomposition
}

\author{
M. Sasaki ${ }^{1,2,3}$, T. Kobayashi ${ }^{4}$, R. O. Dendy ${ }^{2,3,5}$, Y. Kawachi ${ }^{6}$, \\ H. Arakawa ${ }^{7}$, S. Inagaki ${ }^{1,2}$ \\ ${ }^{1}$ Research Institute for Applied Mechanics, Kyushu University, Kasuga \\ 816-8580, Japan \\ ${ }^{2}$ Research Center for Plasma Turbulence, Kyushu University, Kasuga \\ 816-8580, Japan \\ ${ }^{3}$ Centre for Fusion, Space and Astrophysics, Department of Physics, \\ Warwick University, Coventry CV4 7AL, United Kingdom \\ ${ }^{4}$ National Institute for Fusion Science, Toki 509-5292, Japan \\ ${ }^{5}$ CCFE, Culham Science Centre, Abingdon, Oxfordshire OX14 3DB, \\ United Kingdom \\ ${ }^{6}$ Interdisciplinary Graduate School of Engineering Sciences, Kyushu \\ University, Kasuga 816-8580, Japan \\ ${ }^{7}$ Institute of Science and Engineering, Academic Assembly, Shimane \\ University, Matsue 690-8504, Japan \\ E-mail: sasaki@riam.kyushu-u.ac.jp
}

\begin{abstract}
A method to quantify the energy transfer among turbulent structures using singular value decomposition (SVD) is presented. We apply the method to numerical turbulence data obtained from a global plasma simulation using the Hasegawa-Wakatani fluid model, in which the Kelvin-Helmholtz instability plays a dominant role. Using the SVD method, the electrostatic potential is decomposed into a background potential deformation, a zonal flow, a coherent mode and an intermittent structure. Thus there are four key structures, as distinct from the three found in conventional theory. The kinetic energy of each structure is evaluated, and the limit cycle among them is obtained. In the limit cycle, an abrupt change of the background is found to be synchronised with the period of the zonal flow. The energy transfer function of each turbulence structure, which is defined on the basis of a vorticity equation, is evaluated. This then provides physical understanding of how the limit cycle is sustained by dynamical changes in the energy transfer among structures over the its period. In addition, it is shown that the abrupt deformation of the background is caused by the nonlinear self-coupling of the intermittent structure.
\end{abstract}




\section{Introduction}

Magnetically confined plasmas are nonequilibrium open systems, in which nonlinear structures are observed to arise spontaneously on the largest accessible scales. These structures typically feed on microturbulence [1], and include zonal flows [2], bulk intrinsic toroidal rotation [3], and transport barriers that can arise both within the plasma [4] and at its boundary [5]. This phenomenology is of great practical importance, because the transport of energy, momentum and particles across the plasma is conditioned by, and self-consistent with, the excitation and continuing existence of the largescale nonlinear structures [6]; the latter are often referred to as secondary structures, to distinguish them from the primary microturbulence. Understanding and predicting this phenomenology, and its consequences for the local and global behaviour of the plasma, is greatly assisted if techniques applicable to low-dimensional dynamical systems [7] can be deployed. This approach requires quantitative spatio-temporal characterisation of the salient nonlinear structures and of the microturbulence, together with the couplings between them.

A pre-requisite for this approach is to have a reliable means to identify such structures, by extracting them from experimental data and from the outputs of largescale numerical simulations. Indeed it can be a non-trivial task to determine how many distinguishable nonlinear structures are present. This is important both for quantitative characterisation of the data, and to motivate or validate the adoption of lowdimensional analytical models in the predator-prey genre for turbulence phenomenology, see for example Refs.[2], [8]-[9]. Notable current methods include singular value decomposition (SVD) [10, 11], [12, 13, 14, 15, 16] and dynamical mode decomposition (DMD) $[17,18,19]$. These methods have been widely applied in plasma physics, for example to the extraction of fluid turbulent structures [20] and the visualization of phase space structures [21]. In the DMD method, the time evolution of the field is assumed to be exponential, hence the computational cost is low. However, while the exponential assumption can work well to characterise system dynamics over relatively short timescales, beginning at any instant, it is not well adapted to system dynamics that incorporate limit cycle oscillations (LCOs) [22], which are a focus of the present paper. In plasma physics, LCOs are often observed near the threshold for local or global transitions that may also involve structure formation. Examples span electric field pulsations [23], phenomenology near the threshold for L-H transitions in global energy confinement [24, 25, 26], and edge localized modes [27]. These LCOs may significantly affect overall plasma performance, so that understanding their underlying physics is important. While the assumption of exponential time dependence in DMD treatments can be mitigated to some extent [17], DMD is not well adapted to LCOs where, as in the present paper, there is a cyclic rise and fall in the intensity of turbulence, of turbulence-driven structures, and of the coupling between them.

On the other hand, the SVD method makes no prior assumption regarding time evolution, so that the extraction of, for example, frequency chirping and phase modulation becomes possible. SVD is based on functional decomposition with respect 
to orthogonal basis functions which are, by construction, uncoupled to each other. This greatly assists the evaluation of the intrinsically nonlinear forces and fluxes, which are expressed in relation to the decomposed structures, and which drive the fluctuations.

Here we apply the SVD method to a dataset obtained from a specific global simulation [28] of a magnetised cylindrical plasma based on the nonlinear HasegawaWakatani coupled two-fluid model [29] for density and electrostatic potential. In this simulation, it has been found [28] that LCOs arise spontaneously, with phenomenology dominated by the Kelvin-Helmholtz (KH) instability [30]. Using the SVD method, the electrostatic potential is decomposed into the background flow, the zonal flow, a coherent mode, and an intermittent structure. We find that the LCO between these turbulent structures is synchronised with the periodicity of the zonal flow, and involves an abrupt change in the background flow. An energy transfer function based on the vorticity equation is introduced. This enables us to evaluate the cyclic dynamics of how the energy transfer among the turbulent structures changes during the LCO. The rest of the paper is organised as follows. Section 2 introduces the simulation dataset used in this study. In section 3, we describe how SVD is used to extract the spatiotemporal pattern of the turbulence. The dynamics of the energy transfer among the structures is evaluated in section 4. A summary is given in section 5 .

\section{Abrupt change of background and turbulence}

We first introduce the dataset to which the SVD method will be applied. It relates to turbulent phenomena that arise in a global three-dimensional fluid simulation of a magnetised cylindrical plasma, based on the Hasegawa-Wakatani model [31]. The magnetic field, which is in the axial direction, is spatially uniform and constant. The turbulence and background plasma profiles are self-consistently calculated by the fluxdriven simulation with a set of parameters similar to those in basic experiments in linear devices [28]. A radially inhomogeneous background flow is driven by introducing a vorticity source, such that the plasma is linearly unstable against the KH instability. The fully nonlinear evolution of the $\mathrm{KH}$ instability is captured by the simulation. In cases with a weak vorticity source, stationary nonlinear saturation is obtained. Above a certain threshold for the intensity of the vorticity source, LCO occurs [28], and we focus on this regime in the present study. Details of simulation condition and the basic characteristics of the fluctuations are given in [28].

Figure 1(a) shows a snapshot of the fluctuating component of the electrostatic potential. Noting that the electrostatic potential is intrinsically related to the $\mathrm{E} x \mathrm{~B}$ bulk plasma flow, hereafter we use the terms potential or flow interchangeably, depending on the context. Here the fluctuation is translationally invariant along the axis of the cylinder; with respect to azimuthal angle, the component $m=1$ is dominant, where $m$ is the azimuthal mode number. This is characteristic of the nonlinear KH instability in the scenario considered [30]. The spatial pattern is dynamically modulated under the LCO, whose period is much longer than that of azimuthal rotation; further detail 

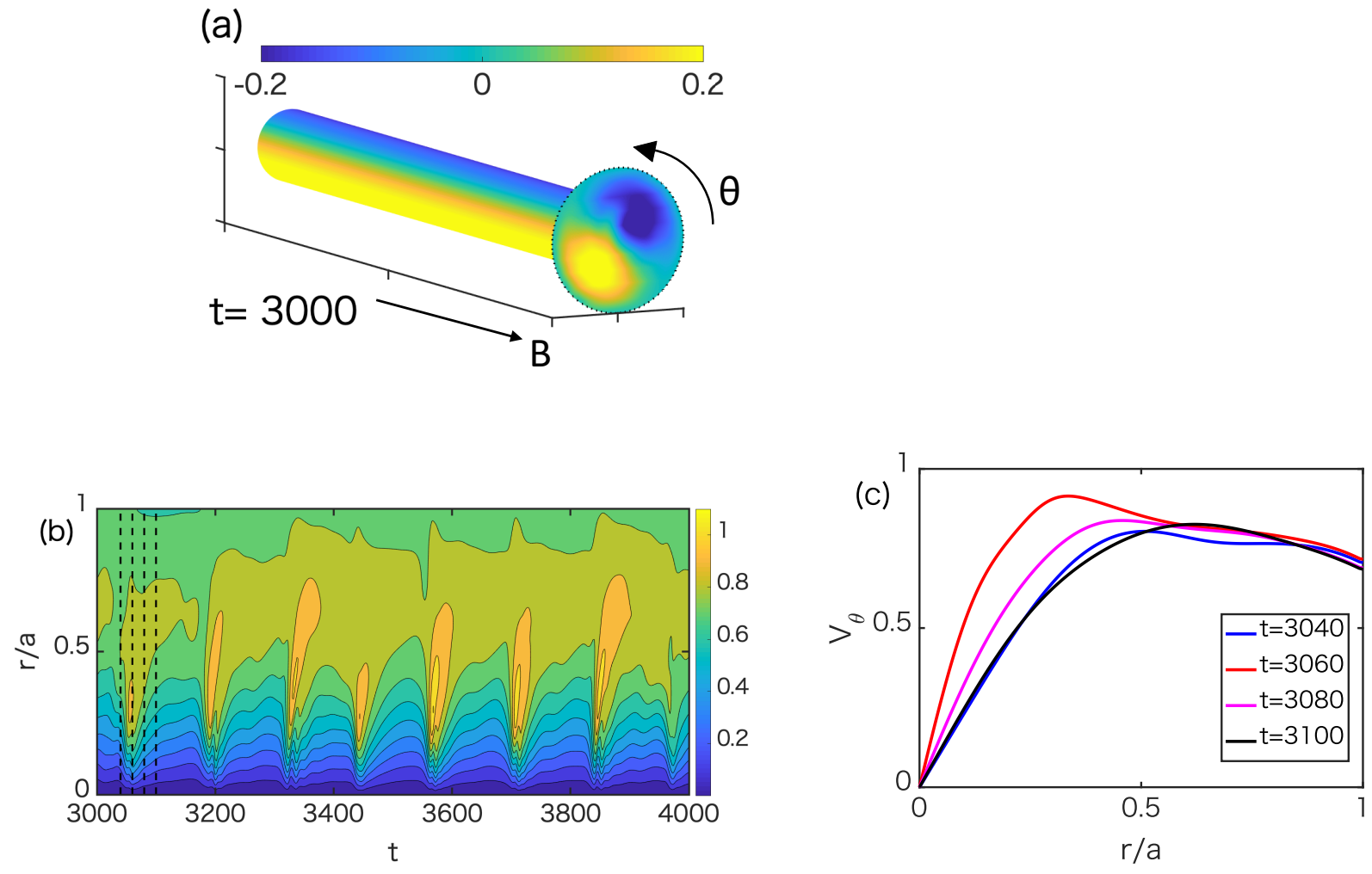

Figure 1. (a) Snapshot of three dimensional pattern of the electrostatic potential fluctuation at $t=3000$. (b) Time evolution of the background azimuthal flow, calculated from the electrostatic potential with $m=n=0, V_{\theta}=\partial_{r} \phi_{0,0}$, where $m$ and $n$ are the azimuthal and axial mode numbers, respectively. (c) Radial profile of background flow at $t=3040,3060,3080$ and 3100, where these times are shown in the black dashed lines in Fig. 1(b).

is given in Ref. [28]. Figure 1(b) shows the time evolution of the background flow, calculated from the electrostatic potential with $m=n=0, V_{\theta}=\partial_{r} \phi_{0,0}$, where $m$ and $n$ are the azimuthal and axial mode numbers, respectively. The radial profiles of $V_{\theta}$ at different times are shown in Fig. 1(c). The flow curvature, which is the driving force for the KH instability, becomes strong and weak in a LCO manner. The magnitude of the velocity variation under LCO is approximately $30 \%$.

Figure 2(a) shows the time evolution of a Fourier power spectrum with respect to azimuthal modes of the electrostatic potential. The mode spectrum for azimuthal modes up to $m=15$ shows that the LCO has a period $T_{\text {period }} \sim 150$. To further illustrate the temporal dynamics of the background potential, we construct an empirical instantaneous growth rate from the simulations, using the definition $\gamma(t)=\partial_{t} \ln E_{0,0}$, where $E_{0,0}$ is the kinetic energy of the background flow, calculated from the background potential. That is, $E_{0,0}=\int\left|\nabla_{\perp}\langle\phi\rangle\right|^{2} d^{3} x$, where $\langle\cdots\rangle$ denotes the zonal average (average in the azimuthal and axial directions). The time evolution of $\gamma(t)$ is plotted in Fig. 2(b), showing a sequence of rapidly rising spikes which are characteristic of this type of abrupt phenomenon [32]. 


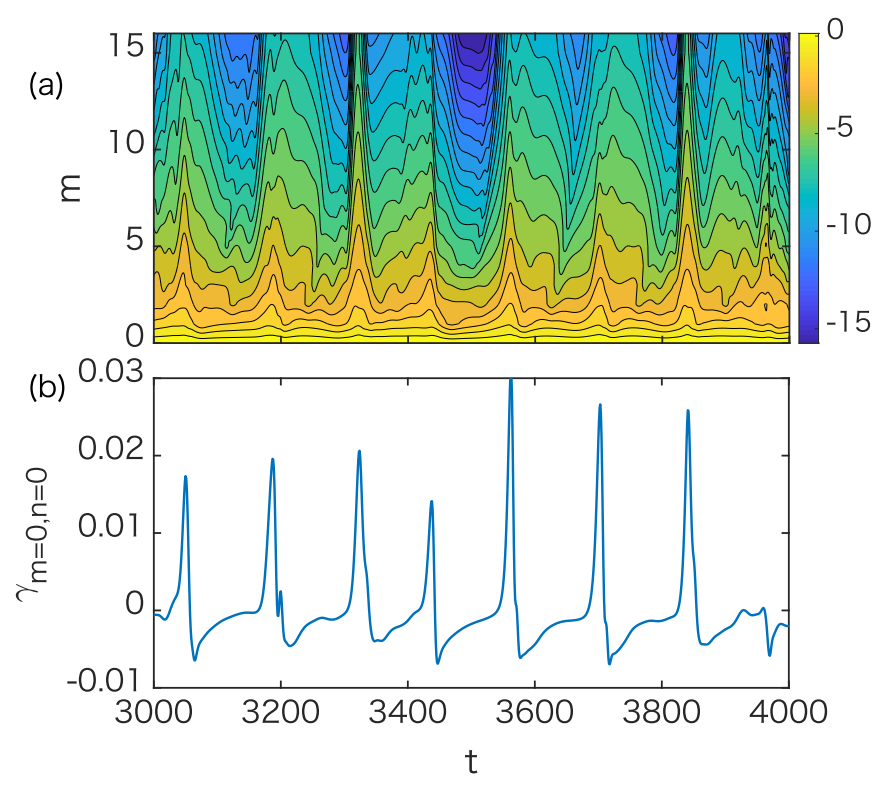

Figure 2. Time evolution of: (a) the azimuthal mode number spectrum of the electrostatic potential in a logarithmic scale; (b) the empirical instantaneous growth rate of the kinetic energy of the background flow, $\gamma_{m=0, n=0}=\partial_{t} \ln E_{0,0}$.

\section{Evaluation of energy transfer by SVD}

Let us now apply the SVD method to the dataset described in the previous section, so as to extract the dominant spatiotemporal structures and evaluate the energy transfer among them. We shall also examine the physical origin of the abrupt increases of the background flow noted in Fig.2, and identify the role of each structure in the LCO.

Because the $\mathrm{KH}$ turbulence is purely two-dimensional, we analyse the electrostatic potential $\phi(r, \theta, t)$; here $\phi(r, \theta, t)$ includes both the fluctuations and the background deformation. We note that the time averaged component is extracted before the analysis. Using the SVD method, $\phi(r, \theta, t)$ is decomposed as

$$
\phi(r, \theta, t)=\sum_{j} s_{j} \Psi_{j}(r, \theta) h_{j}(t),
$$

Here, in relation to the $j$ th mode: $\Psi_{j}(r, \theta)$ describes the mode's spatial structure; $h_{j}(t)$ captures its temporal evolution; and $s_{j}$ is its singular value, that is, the weight that defines the relative importance [10] of the mode's contribution to $\phi(r, \theta, t)$. We emphasise that the functional form of each $\Psi_{j}$ is entirely dictated a posteriori by the simulation outputs, without any prior assumptions. In Appendix A, we outline how to perform the SVD method to the dataset observed in two-dimensional system. Figure 3 shows the dominant structure functions $\Psi_{j}$, and their time evolution $h_{j}$. These are the top nine modes, in descending order of their singular values $s_{j}$. We note that the two-dimensional pattern of $\phi(r, \theta, t)$ dynamically changes in time, as shown in Fig. 2 for Fourier space, or Fig. 1 of [17] for real space. The nine spatial structure functions shown in the left panel of Fig. 3 capture background profile deformation (modes 1 and 4) and turbulent 
structures and fluctuations (the remaining modes). Here the mode ID numbers $1,2, \cdots 9$ are in descending order of the singular values $s_{j}$. These are the dominant modes in this SVD, as can be seen from the right panel of Fig. 3, where values of $s_{j}$ are plotted on a logarithmic scale for modes up to the hundredth. In the present study, we truncate the SVD modes at the fortieth, for which the energy transfer can be calculated within acceptable accuracy. Convergence checks in relation to mode truncation are discussed in Appendix B.

One can see from Fig. 3 that there are several paired modes whose spatial structures are similar and whose singular values are nearly the same, which differ only in their phase. Examples of paired modes are: $\mathrm{ID}=2,3, \mathrm{ID}=5,6$, and $\mathrm{ID}=7,8,9$. These are complex conjugate pairs, which in combination represent the azimuthal propagation, akin to the combination of sine and cosine components in Fourier mode decomposition. Thus, the summation of the pair should be treated as a single structure. There are also unpaired modes, for example modes $\mathrm{ID}=1$ and $\mathrm{ID}=4$, which are almost azimuthally homogeneous and do not propagate in the azimuthal direction. The pairing of modes can be demonstrated from their envelopes calculated from $h_{j}$ as shown in the middle panel of Fig. 3. The envelopes, delineated in black, change slowly in time; here the envelopes represent $\sqrt{h_{2}^{2}+h_{3}^{2}}($ for $\mathrm{ID}=2,3), \sqrt{h_{5}^{2}+h_{6}^{2}}($ for $\mathrm{ID}=5,6)$ and $\sqrt{h_{7}^{2}+h_{8}^{2}+h_{9}^{2}}(\mathrm{ID}=7,8$, $9)$. One can see that the temporal behaviours of modes with $\mathrm{ID}=5-9$ are intermittent (time scales of their growth and decay are comparable to their frequency), while the modes with ID=2-3 are coherent. We wish to capture low-dimensional phenomenology wherever possible, and therefore choose to reduce the number of degrees of freedom by taking a summation from mode $\mathrm{ID}=5$ to $\mathrm{ID}=40$; we then treat the resulting summed entity as a single structure.

The spatiotemporal behaviour of the electrostatic potential is thus decomposed into four structures, $\phi_{A}, \phi_{B}, \phi_{C}, \phi_{D}$, which are defined as follows:

$$
\begin{aligned}
& \phi(r, \theta, t) \approx \sum_{\zeta=A \sim D} \phi_{\zeta}(r, \theta, t), \\
& \phi_{A}(r, \theta, t)=\varphi_{1}(r, \theta, t), \\
& \phi_{B}(r, \theta, t)=\varphi_{4}(r, \theta, t) \\
& \phi_{C}(r, \theta, t)=\varphi_{2}(r, \theta, t)+\varphi_{3}(r, \theta, t), \\
& \phi_{D}(r, \theta, t)=\sum_{j=5}^{40} \varphi_{j}(r, \theta, t),
\end{aligned}
$$

where $\varphi_{j}(r, \theta, t)=s_{j} \Psi_{j}(r, \theta) h_{j}(t)$. Snapshots of each of these four structures at $\mathrm{t}=3200$ are shown in Fig. 4. Mode A corresponds to the background potential deformation; mode $\mathrm{B}$ to the zonal flow; mode $\mathrm{C}$ to the coherent $\mathrm{KH}$ mode; and mode $\mathrm{D}$ to the intermittent structure. Here, we note mode $\mathrm{A}$ and mode $\mathrm{B}$ are distinguished from their radial wavenumber; $k_{r} \leq \pi / a$ (mode $\mathrm{A}$ ) and $k_{r}>\pi / a$ (mode $\left.\mathrm{B}\right)$, where $k_{r}$ is the radial wavenumber of the electrostatic potential with $m=n=0$. We refer to these as extracted structures of the electrostatic potential. The kinetic energy of each structure, 

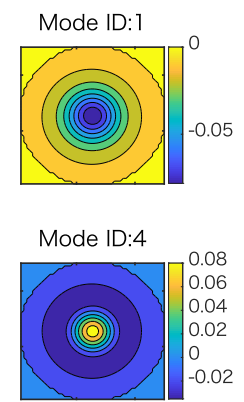

Mode ID:7

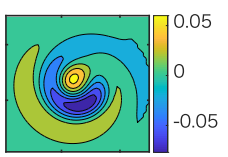

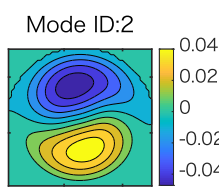
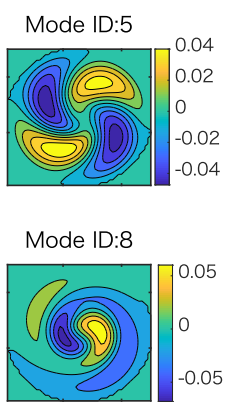
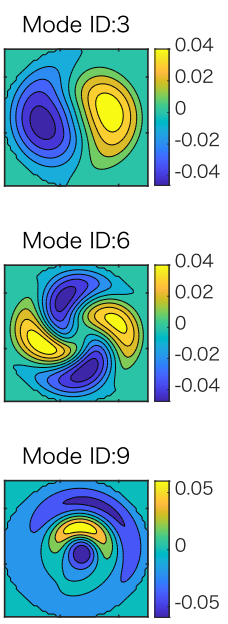

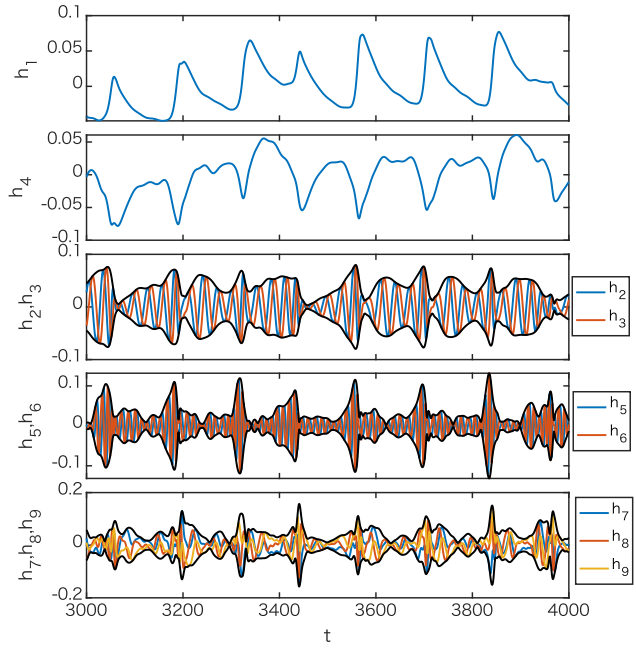

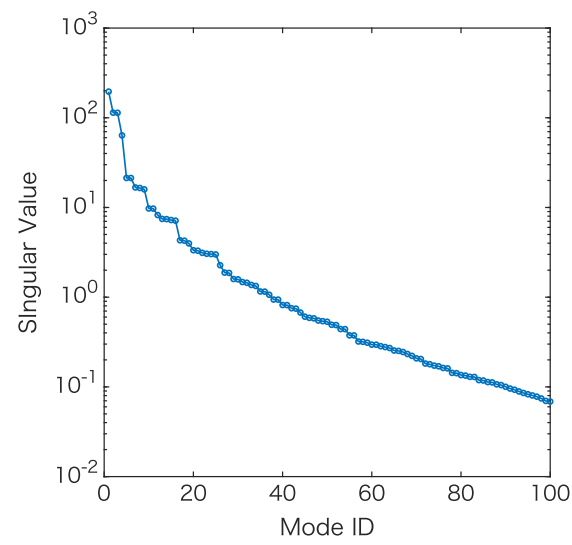

Figure 3. (Left panel) The spatial character of the nine SVD modes that have the largest singular values. (Middle panel) The time evolution of the SVD modes, characterised by $h_{j}(t)$ in Eq. (1). (Right panel) Singular values of the first twenty SVD modes, plotted on a logarithmic scale.

$E_{\zeta}$ an be evaluated from the definition

$$
E_{\zeta}=\frac{1}{2} \int\left|\nabla_{\perp} \phi_{\zeta}\right|^{2} d^{3} x, \quad(\zeta=A \sim D) .
$$

The time evolution of the kinetic energy of each structure is illustrated in Fig. 5(a). Information about the relative phase of the time-evolving kinetic energy in pairs of modes can also be inferred from the phase plots in the right panel of Fig.5. The process for constructing these plots is identical to that for Lissajous figures. While, strictly, the latter term applies to superimposed simple harmonic motions, we use it more colloquially here and in Figs. 8 and 9. Lissajous figures (in this sense) are widely used to characterise strongly nonlinear structures in plasmas, see for example the treatment of heat pulse propagation in the LHD heliotron-stellarator in Figs.1,3,5,6 and 7 of Ref.[33] and Fig.7 of Ref.[34].

The behaviour of the energy that is evident in Fig. 5 is similar to that extracted by using the DMD method, see in particular Figs. 4 of Ref.[17]. The background potential 

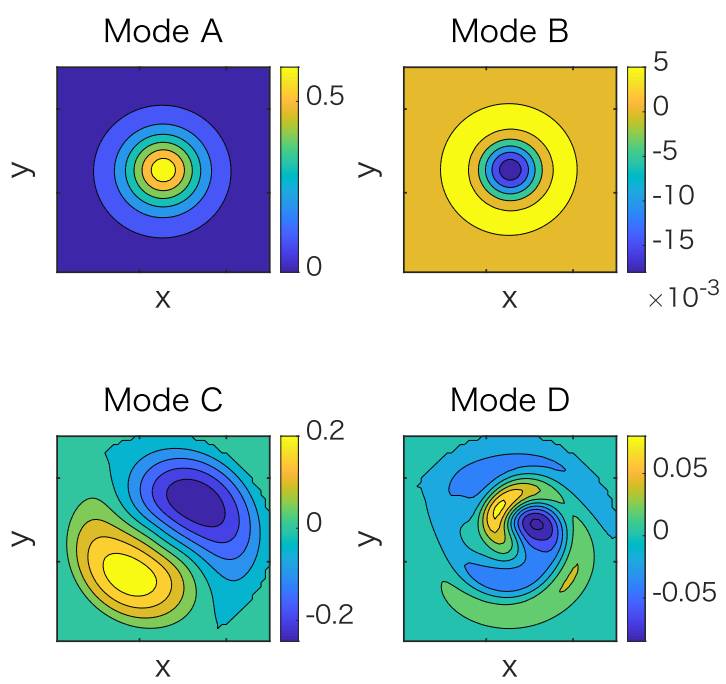

Figure 4. Dominant turbulent structures extracted by summation and truncation of the modes in Fig. 3. Mode A is invariant against azimuthal rotation, and the dominant component of Mode B is an azimuthally symmetric zonal pattern. Modes C and D are the turbulent structures.

(mode $\mathrm{A}$ ) and the coherent $\mathrm{KH}$ mode $\mathrm{C}$ compete with each other. When the magnitude of mode $\mathrm{C}$ increases to a certain threshold, the zonal flow mode $\mathrm{B}$ is excited together with the intermittent structure mode D. After the appearance of modes B and D, mode $\mathrm{C}$ decreases and mode A increases. This cycle then repeats - it is an LCO. The period of the LCO is synchronised with the zonal flow oscillation. The time evolution of the local value of the zonal potential (mode $\mathrm{B}$ ) at different radial locations is plotted in Fig. 5(b). The phase of mode $\mathrm{B}$ at $r / a=0.23$ is the reverse of the phase at $r / a=0.6$. The plot of the time-evolution of the potential at these two radial locations exhibits standing-wave-like structure. Successive zero-crossings - that is, phase reversal events are synchronous with successive cycles of the LCO, where the frequencies of the LCO and the zonal flow are similar and the phase relation between the zonal flow and the LCO does not vary over time. The zonal flow mode B always peaks sharply just before each abrupt increase of the background flow mode A, and the phase of mode B reverses after the burst of mode A.

\section{Energy transfer dynamics among SVD modes}

The energy transfer among the structures extracted in the preceding section can be characterised in terms of an energy transfer function that is based on the vorticity equation. The kinetic energy evolution equation can be derived by multiplying both sides of the vorticity equation by $\phi$ and performing a spatial integration. The details of the derivation is described in Appendix C. Here, $\phi$ is decomposed into the SVD structures from mode A to mode D as in Eq. (2a). The kinetic energy of Mode $\zeta$ can 

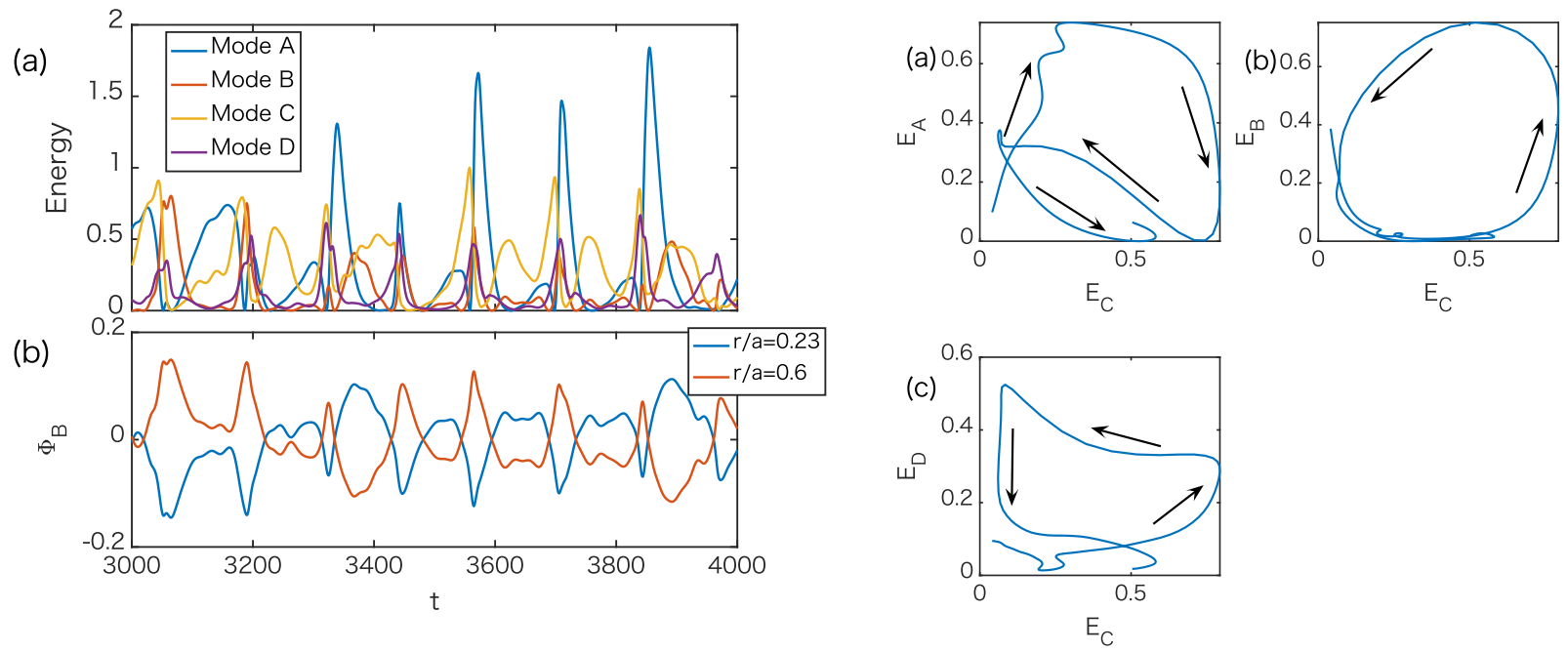

Figure 5. Characterising limit cycle oscillations. Left panel. Time evolution of: (a) the kinetic energy of each of the four structures A to D identified in Fig. 4; (b) the local values of the zonal flow potential (Mode B) at widely separated radial locations $r / a=0.23$ and 0.6 , at angular position $\theta=0$. Right panel. Three Lissajous figures plotting the time evolution of the kinetic energy of the three Modes A, B and D against that of Mode $\mathrm{C}$, during the time interval $3080<t<3250$. The arrows show the direction of time evolution.

be written as $(\zeta=A \sim D)$

$$
\begin{aligned}
& \partial_{t} E_{\zeta}=\sum_{\alpha, \beta=A \sim D} J(\zeta \mid \alpha, \beta)-2 \nu E_{\zeta}-\int \phi_{\zeta} S d^{3} x+F_{\zeta}, \\
& J(\zeta \mid \alpha, \beta)=\int \phi_{\zeta}\left[\phi_{\alpha}, \nabla_{\perp}^{2} \phi_{\beta}\right] d^{3} x,
\end{aligned}
$$

where the square brackets in Eq. (4b) denote the convective derivative defined as $\left[\phi, \nabla_{\perp}^{2} \phi\right]=r^{-1}\left(\partial_{r} \phi \partial_{\theta} \nabla_{\perp}^{2} \phi-\partial_{r} \nabla_{\perp}^{2} \phi \partial_{\theta} \phi\right)$, and $\nu$ and $S$ are the ion-neutral collision frequency and the vorticity source, respectively. The term $F_{\zeta}$ is the other nonlinearities introduced in Appendix C. In this study, we focus on the term related to the convective nonlinearity. The energy transfer function, $J(\zeta \mid \alpha, \beta)$, quantifies the energy flow to the mode labelled $\zeta$ that arises from nonlinear coupling between the modes labelled $\alpha$ and $\beta$. Positive (or negative) $J(\zeta \mid \alpha, \beta)$ contributes to driving (or damping). The terms in the RHS of Eq. (4a) are linear, except for the energy transfer term. In the present SVD context, Eq. (4a) can be viewed as a natural extension of the energy transfer equation in Fourier space [35, 36]. It is well adapted to understanding the energy interaction among the turbulent structures identified using SVD, which contain multiple Fourier components. In the absence of such structures, a Fourier-based approach to energy transfer would suffice for zonal flows, which can be described by a single Fourier component. It follows that the evidently nonlinear features of the system dynamics, for example those shown in Fig. 5, must stem from the energy transfer among the turbulent structures. In order to understand the underlying physics of the system, it is therefore necessary to examine the behaviour of the energy transfer function in greater detail. 
As a preliminary, we note that $J(\zeta \mid \alpha, \beta)$ is not symmetric with respect to $\alpha$ and $\beta$. Given the four structures A to D, there are thus sixteen relevant mode pairings. Table 1 specifies how we label ("Coupling ID") these pairings using the first sixteen integers. We note that the SVD method does not involve well-defined matching criteria with respect to frequency or wavenumber because, in general, SVD modes contain multiple Fourier modes. In the present paper, mode $\mathrm{D}$ is a good example of this.

Figure 6 seeks to capture the temporal variation of $J(\zeta \mid \alpha, \beta)$ for each structure A to $\mathrm{D}$, resulting from each of the sixteen coupling combinations, from two different perspectives. The four left panels, Fig. 6 (a)-(d), plot the instantaneous values that are taken by each $J(\zeta \mid \alpha, \beta)$ at each unit time-step during the interval $3000<t<4000$ which was also considered in Figs. 2, and 5. For example, Fig. 6(c) shows that for mode $\mathrm{C}$, the four sources/sinks of nonlinear drive that contribute most strongly to energy transfer (both positive and negative) are the mode pairings with coupling ID = 4, 8, 13 and 14. From Table 1, in $(\alpha, \beta)$ terms, these pairings are (A,D), (B,D), (D,A) and $(\mathrm{D}, \mathrm{B})$. Conversely, from Fig. $6(\mathrm{~b})$ it is evident that energy transfer to and from mode $\mathrm{B}$ is dominated by the mode pairing with coupling ID =11, 12, 15, 16 that are $(\alpha, \beta)=(\mathrm{C}, \mathrm{C}),(\mathrm{C}, \mathrm{D}),(\mathrm{D}, \mathrm{C}),(\mathrm{D}, \mathrm{D})$. From Fig. 6(a), one can see that the background flow, mode $\mathrm{A}$, is affected primarily by the self-couplings $(\mathrm{C}, \mathrm{C})$ and $(\mathrm{D}, \mathrm{D})$ and by the pair couplings $(\mathrm{C}, \mathrm{D})$ and $(\mathrm{D}, \mathrm{C})$. There is no coupling between the zonal flow and the background flow, which is because the background flow does not generate the doppler shift for the zonal flow. The background and the zonal flows couple indirectly through the deformation of the fluctuations. We note from Fig. 4 that the azimuthally symmetric background flow is contained in mode $\mathrm{A}$ and partly in mode $\mathrm{B}$. This flow is driven by the vorticity source. It follows that net negative nonlinear energy transfer to mode A acts to relax the background flow. In contrast, for mode $\mathrm{C}$ and mode $\mathrm{D}$, nonlinear damping due to self-coupling is found to be weak, in that the contributions from the coupling $\mathrm{ID}=11$ for mode $\mathrm{C}$ and 16 for mode $\mathrm{D}$ are small. The four right panels, Fig. 6(e)(h), plot the explicit time evolution of $J(\zeta \mid \alpha, \beta)$ on a colour scale, for the four modes (one panel per mode) in relation to the sixteen mode pairings. The energy transfers among the structures are strongly non-time-stationary: the discrete bursts of activity correlate necessarily with those in Fig. 5, which align with the LCO period, as discussed previously. In the following, in order to reduce the number of coupling combinations, we treat the couplings of mode- $\alpha$ with mode- $\beta$ and of mode- $\beta$ with mode- $\alpha$ together as $J(\zeta \mid \alpha, \beta)+J(\zeta \mid \beta, \alpha) \rightarrow J(\zeta \mid \alpha, \beta)$.

The energy transfer functions for each structure during a single LCO period, $3120<t<3220$, are illustrated in Fig. 7, where only the most important couplings are shown. There are three phases, because the energy flow pattern changes significantly between the first half $(3120<t<3180)$, the middle regime $(t \sim 3190)$, and the second half $(3200<t<3220)$. This pattern corresponds to the growth, saturation and damping of the zonal flow, mode B. The energy transfer to the zonal flow changes its sign at $t \sim 3190$, at which time the zonal flow peaks in energy. This saturation is caused by the reversal in energy transfer $J(B \mid C, D)$. This implies the coupling $C+D$ 
is important for the nonlinear saturation of the zonal flow. Simultaneously, there is an abrupt onset to the nonlinear drive of the background flow (mode A), due to the nonlinearity of mode D, as shown in Fig. 7(b), see Fig. 7(a); the energy of mode A, previously decaying, starts to increase.

The $x$-axis of all three panels of Fig. 8 is $J(C \mid A, C)$. This is chosen because, in general, $J(\zeta \mid A, \zeta)$ represents the generalised linear drive of the mode labelled $\zeta$ by the $\mathrm{KH}$ instability, which arises from deformation of the background potential mode A. This drive would be linear, notwithstanding its apparently nonlinear mathematical character, in an ordering scheme where mode $\mathrm{A}$ is zeroth order while the other modes are order-epsilon. In Fig. 8 the focus is thus on the different nonlinear drives of the coherent $\mathrm{KH}$ mode $\mathrm{C}$, in relation to the linear drive. The linear drive for the coherent $\mathrm{KH}$ mode $\mathrm{C}$, which corresponds to $J(C \mid A, C)$, changes its role in accordance with the phase of the LCO. It is apparent from Fig. 7(a) that, during the first half of the LCO, mode $\mathrm{C}$ obtains energy from its coupling with the background potential deformation mode $\mathrm{A}$; that is, mode $\mathrm{C}$ is linearly destabilized by the flow inhomogeneity. This phase of the LCO ends at $t \sim 3185$ in Fig. 7 , and this determines the parameter $t_{2}$ which is used throughout Figs. 8 and 9 to denote the end of the first half (blue trace) of the LCO cycle. Thereafter, Fig. 8(a) shows that coupling $J(C \mid B, C)$ of the $\mathrm{KH}$ mode $\mathrm{C}$ with the zonal flow mode $\mathrm{B}$ acts to suppress mode $\mathrm{C}$; this is a back reaction which drives the zonal flow. In the Lissajous figures (Figs. 8 and 9 ), the system trajectory during the three time intervals $3080<t<3185,3185<t<3195$ and $3195<t<3250$ is plotted using blue, red and black lines, respectively. The nonlinear effect of the intermittent structure mode D, characterised by $J(C \mid B, D)$ in Fig. 8(b), has a negligible role during the first half of the LCO. It begins to acquire a small negative value when the linear drive of mode $\mathrm{C}, J(C \mid A, C)$, approaches its maximum value of 0.015 , and then rises abruptly after the onset of the zonal flow (red trace). The plot of $J(C \mid D, D)$ in Fig. $8(\mathrm{c})$ shows that the nonlinear self-interaction of mode $\mathrm{D}$ has a negative effect on mode $\mathrm{C}$ in the first and middle phase of the LCO. At the conclusion of the zonal flow drive, as $J(C \mid A, C)$ declines towards zero, $J(C \mid D, D)$ starts (black trace) to contribute to the drive of mode $\mathrm{C}$. In this way, the nonlinear modifications to mode $\mathrm{C}, J(C \mid B, D)$ and $J(C \mid D, D)$, change their character after the onset of the zonal flow.

The linear drive for the intermittent structure mode $\mathrm{D}$ corresponds to $J(D \mid A, D)$. The Lissajous figures for $J(D \mid \alpha, \beta)$ during the LCO are shown in Fig. 9, and demonstrate that $J(D \mid A, D)$ changes its role in accordance with the phase of the LCO, like $J(C \mid A, C)$ in Fig. 8 [17]. Figure 9(a) shows that for mode D, unlike for mode C, the deformation of the $\mathrm{KH}$ mode $\mathrm{C}$ due to its nonlinear coupling with the background flow mode A contributes to the drive. Deformation of mode $\mathrm{C}$ by the zonal flow $\mathrm{B}$ also affects the energy transfer to Mode D, as can be inferred from Fig. 9(b). This shows a rather abrupt reversal between the first half (blue trace) and second half (red, then black, traces) of the LCO, giving rise to a well-defined figure-8. The nonlinear coupling of Mode D with the zonal flow mode B is shown in Fig. 9(c). The sign of $J(D \mid B, D)$ is always negative, implying that it contributes to the suppression of mode $\mathrm{D}$ throughout 
the LCO. As seen in the Lissajous figure for $J(D \mid C, D)$ in Fig. 9(d), nonlinear coupling with mode $\mathrm{C}$ drives mode $\mathrm{D}$ during the first half (blue trace) of the LCO; damps it during the middle phase (red trace) and much of the final phase (black trace).

The energy flow patterns inferred from Figs. 8 and 9 during a single LCO period are summarized in Fig. 10. We have found that important roles in the sustainment of the LCO are played by the secondary structures, namely the zonal flow mode B and the intermittent structure mode D, notably through their nonlinear coupling with the $\mathrm{KH}$ mode $\mathrm{C}$.

We now perform a quantitative check on our inference that the abrupt energy input to the background flow mode $\mathrm{A}$ at $3185<t<3195$ causes the abrupt increase in the background flow that is shown in Fig. 2. Dividing both sides of Eq. $(4 a)$ by $E_{\zeta}$, an equation for the time constant of mode $\mathrm{A}, \gamma_{A}$, can be formally derived as

$$
\gamma_{A}=E_{A}^{-1} \sum_{\alpha, \beta} J(A \mid \alpha, \beta)-\nu
$$

From Fig. 2, the time constant at the abrupt increase can be estimated as $\gamma_{A} \sim$ $2 \times 10^{-2} \gg \nu=5 \times 10^{-3}$. This value can be explained as $E_{A}^{-1} \sum_{\alpha, \beta} J(A \mid \alpha, \beta) \sim 2 \times 10^{-2}$, where we use $E_{A}=0.5$ and $\sum_{\alpha, \beta} J(A \mid \alpha, \beta) \approx J(A \mid D, D) \sim 0.01$. This tends to confirm that the abrupt increase of the background flow mode $\mathrm{A}$ is realised by the nonlinear self-coupling of the nonlinearly driven intermittent structure D. We note that a strong role for the zonal flow mode B in the abrupt change of the background flow is also implicit. This is because, as we have already shown, deformation of modes $\mathrm{C}$ and $\mathrm{D}$ by mode $\mathrm{B}$ abruptly alters the characteristics of nonlinear energy transfer involving modes $\mathrm{C}$ and D.

\section{Discussion and Conclusions}

Using the SVD method, the global phenomenology of the simulated turbulent plasma has been converted into a low-dimensional dynamical system. The electrostatic potential is decomposed into four elements, which are apparently the minimal set: the deformation of the background; the zonal flow; the coherent KH mode; and the intermittent structure. The kinetic energy content of these four modes is found to exhibit an LCO, in which an abrupt change of the background potential is synchronized with the zonal flow period.

Wider application of the SVD method, as presented here, to experimental data from plasmas should be relatively straightforward. While there is no constraint on the number of measurement points in space and time, we note that simultaneous measurements

are necessary to construct the spatiotemporal information matrix, $\Phi\left(x_{i}, t_{j}\right)$, which is introduced in Appendix A. Many typical plasma diagnostics, such as fast camera imaging, Langmuir probes, reflectometers, heavy ion beam probes, beam emission spectroscopy, and tomography, yield datasets which appear suitable for the SVD method. Turning specifically to cylindrical plasmas of the kind considered here, we anticipate that the four key structures identified in the present paper will again be found in future experiments. 

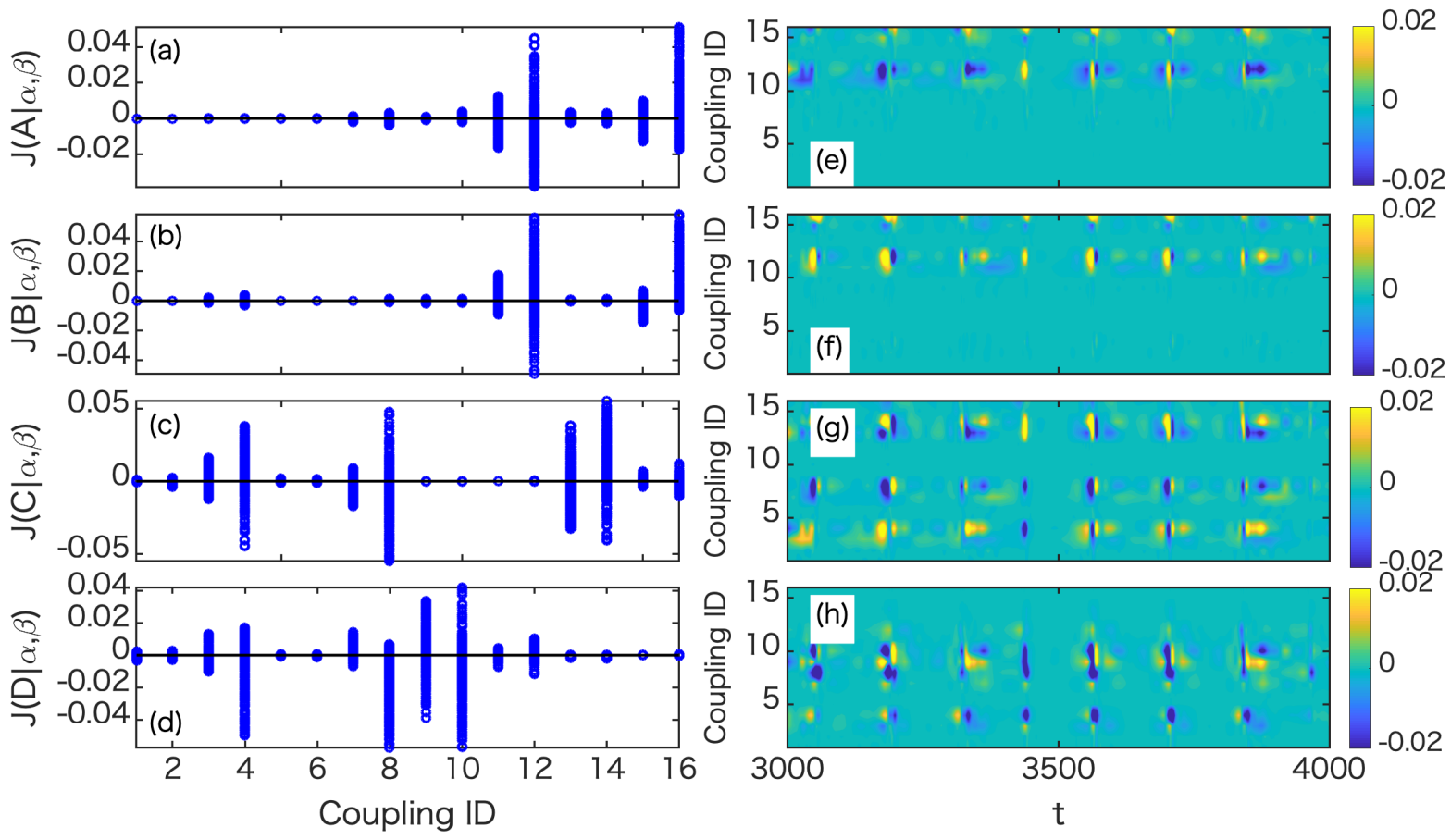

Figure 6. (a)-(d): Time variation of the energy transfer functions for each structure. (e)-(h): Temporal evolution of the energy transfer functions for each structure, where (e) $J(A \mid \alpha, \beta)$, (h) $J(B \mid \alpha, \beta),(\mathrm{g}) J(C \mid \alpha, \beta)$, and (h) $J(D \mid \alpha, \beta)$, where $\alpha+\beta$ denotes the coupling between mode $\alpha$ and mode $\beta$, in the order corresponding to Eq.(4b).

The SVD approach has enabled us to quantify the mutiple nonlinear energy flows that underly this LCO. We first construct and evaluate the energy transfer function for each turbulence structure, based on a vorticity equation. This then provides physical understanding of how the LCO is sustained by dynamical changes in the energy transfer among structures over the LCO period. In summary, coupling between the coherent mode and the intermittent structure drives the zonal flow, which provides resilience for the background potential, which causes the abrupt deformation of the background.

In some previous theoretical approaches, the LCO considered here is often described in terms of a predator-prey model [37] that involves only three elements, namely the background, the zonal flow and microturbulence. Thus, the results in the present paper imply that this conventional model is incomplete. Furthermore, we have found that the hitherto neglected intermittent structure plays crucial roles in the dynamics of the system. The important role of the intermittent structure in the abrupt change in transport has also been identified experimentally [38].

In addition, we have found that the zonal flow oscillation is synchronised with the LCO. The physical mechanism that determines the frequency of the zonal flow remains an unresolved theoretical issue. In the context of the present work, we note that in basic experiments, the excitation of an intermittent structure, referred to as "splash", has been observed to be synchronized with the zonal flow oscillation [39, 40]. This 


\begin{tabular}{cc}
\hline Coupling ID & Combination of structures \\
\hline 1 & $A+A$ \\
2 & $A+B$ \\
3 & $A+C$ \\
4 & $A+D$ \\
5 & $B+A$ \\
6 & $B+B$ \\
7 & $B+C$ \\
8 & $B+D$ \\
9 & $C+A$ \\
10 & $C+B$ \\
11 & $C+C$ \\
12 & $C+D$ \\
13 & $D+A$ \\
14 & $D+B$ \\
15 & $D+C$ \\
16 & $D+D$ \\
\hline
\end{tabular}

Table 1. List of coupling IDs. Each number denotes the combination of structures which contribute to the coupling.

tendency is similar to the behaviour of the intermittent mode D in the present study. This motivates our conjecture that the generation and annihilation of the intermittent structure, together with the deformation of the background flow, may determine the frequency of the zonal flow.

In conclusion, the SVD approach has provided significant new physical insights into the phenomenology of this classical nonlinear KH plasma system.

\section{Acknowledgements}

We acknowledge useful discussions with S. Maeyama of Nagoya University and with O. Samant. This work was partly supported by a grant-in-aid for scientific research of JSPS, Japan (16K18335, 17H06089, 16H02442, 15H02155, 15H02335, 18K03578), the collaboration programs of NIFS (NIFS18KNST137, NIFS19KNST151), Asada Science Foundation, Progress 100 of Kyushu University (NB80645028), and the RIAM of Kyushu University. This work was carried out partly within the framework of the EUROfusion Consortium and has received funding from the Euratom research and training programme 2014-2018 and 2019-2020 under grant agreement No 633053. The work received support from the RCUK Energy Programme grant no. EP/T012250/1. The views and opinions expressed herein do not necessarily reflect those of the European Commission. 


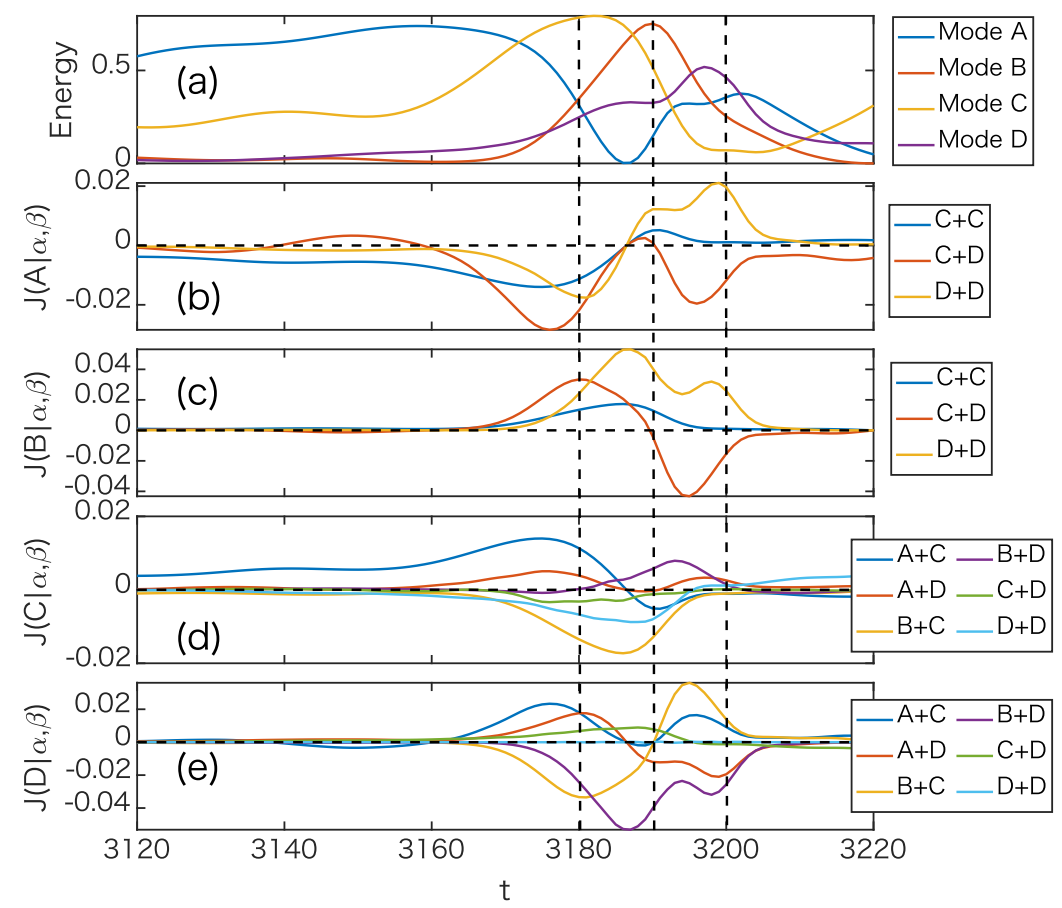

Figure 7. Time evolution of (a) kinetic energy and (b)-(e) energy transfer function for each structure. Only the energy transfer functions for the most important nonlinear couplings are shown.

(a)

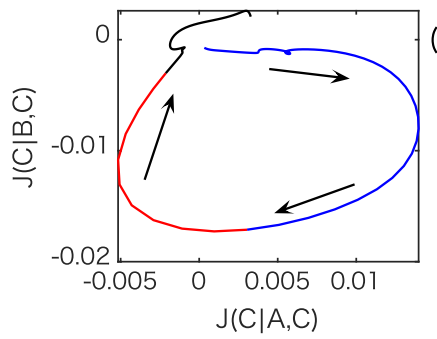

(c)

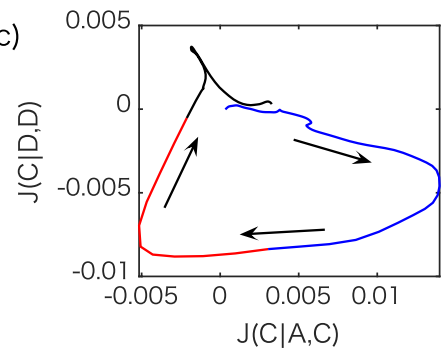

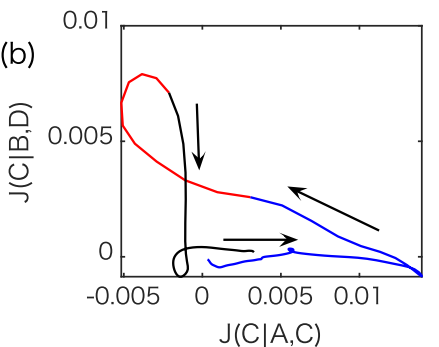

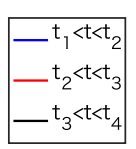

Figure 8. Lissajous figures for the three dominant energy transfer functions for the $\mathrm{KH}$ mode $\mathrm{C}, J(C \mid \alpha, \beta)$, where the arrows show the direction of the time-evolution. Here, $t_{1}=3080, t_{2}=3185, t_{3}=3195$ and $t_{4}=3250$.

\section{Appendix A. SVD analysis in two-dimensional system}

Here we briefly explain how to perform SVD analysis to a set of spatio-temporal data in two-dimensional system. The spatio-temporal data in 2D system can be obtained in 

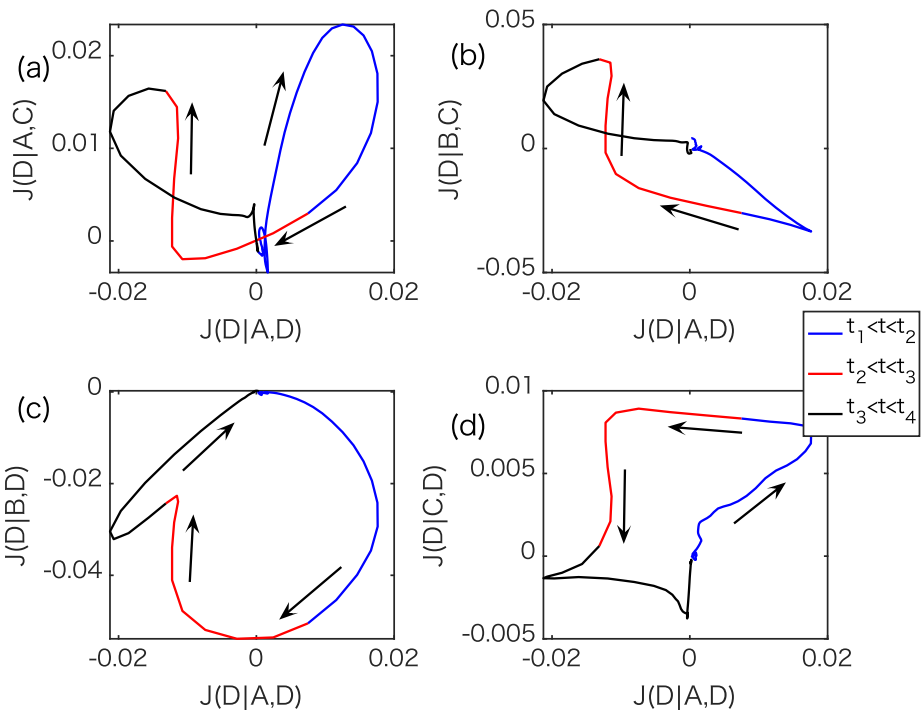

Figure 9. Lissajous figures for the four dominant energy transfer function for the intermittent mode $\mathrm{D}, J(D \mid \alpha, \beta)$, where the arrows show the direction of the evolution. Here, $t_{1}=3080, t_{2}=3185, t_{3}=3195$ and $t_{4}=3250$.

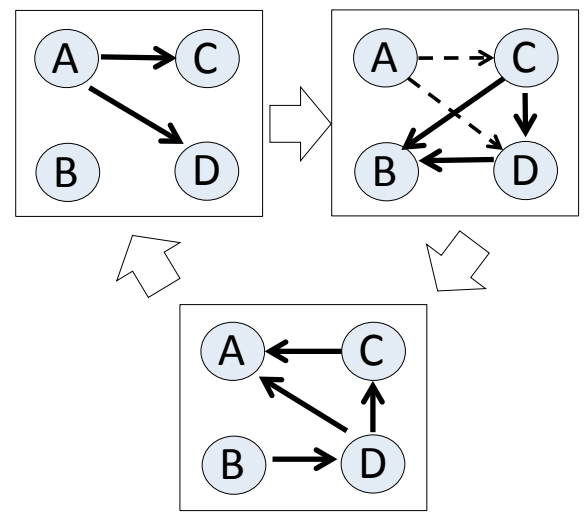

Figure 10. Schematic view of the energy flow among turbulent structures during the limit cycle oscillation, where A-D in the circles denote the Mode A-D, respectively, and the arrows show the directions of the energy flow. The patten of the energy flow changes during one period of the LCO.

a three-dimensional array, $\phi\left(x_{1}, x_{2}, t\right)$, where $x_{j}$ is the spatial coordinate. The size of this array is $M_{1} \times M_{2} \times N$, where $M_{j}$ and $N$ are the number of measurement points regarding $x_{j}$ and time, respectively. In order to perform the SVD, the three-dimensional array is converted to two-dimensional array, $\phi\left(x_{1}, x_{2}, t\right) \rightarrow \Phi(X, t)$, where the size of the matrix is $M \times N$, where $M=M_{1} M_{2}$. Then, the spatio-temporal information matrix, whose component is $\Phi\left(X_{i}, t_{j}\right)=\Phi_{i j}$, can be generally decomposed into $\Phi_{i j}=U \Sigma V^{T}$, where $U$ and $V$ are $M$-th and $N$-th order orhogonal matrix, and $\Sigma$ is $M \times N$ diagonal 
matrix. This expression can be rewritten by

$$
\Phi=\sum_{i=1}^{p} s_{i} \boldsymbol{u}_{i} \boldsymbol{v}_{i}^{T},
$$

where $\boldsymbol{u}_{i}$ and $\boldsymbol{v}_{i}$ are the column vector of $U$ and $V$, respectively, and $s_{i}$ is the component of $\Sigma$. The vectors $\boldsymbol{u}$ and $\boldsymbol{v}$ correspond to spatial and temporal structures, respectively. Then, one can obtain the $2 \mathrm{D}$ pattern by re-converting $X \rightarrow\left(x_{1}, x_{2}\right)$.

Care is needed when spatiotemporal data, expressed with respect to cylindrical coordinates, is decomposed into SVD modes. At first sight, it appears that the orthogonality condition for SVD modes should be given by

$$
\int \phi_{\zeta}(r, \theta) \phi_{\zeta^{\prime}}(r, \theta) d r d \theta=\delta_{\zeta, \zeta^{\prime}},
$$

where $\phi_{\zeta}$ denotes the SVD mode that has mode ID $\zeta$. We note that there is no apparent requirement for a Jacobian $r$ in the integration with respect to the independent variables $r$ and $\theta$ in Eq.(A2). However, when analysing the energy transfer, the spatial integral $\int \cdots r d r d \theta$ is performed. This is necessary for computational accuracy in the context of an intrinsically cylindrical plasma system. It then appears that, for consistency, the SVD orthogonality condition also needs to incorporate a Jacobian $r$, whereas Eq.(A.2) does not.

This conundrum is resolved by defining SVD orthogonality with respect to a Cartesian coordinate system $(x, y)$. On transforming to cylindrical coordinates, this SVD orthogonality condition becomes

$$
\int \phi_{\zeta}(r, \theta) \phi_{\zeta^{\prime}}(r, \theta) r d r d \theta=\delta_{\zeta, \zeta^{\prime}} .
$$

instead of Eq.(A.2). In the present study, we compute the SVD in Cartesian coordinates, after transforming $\phi(r, \theta, t)$ to $\phi(x, y, t)$. This implicitly guarantees the orthogonality condition Eq.(A.3), and is consistent with energy transfer calculations that are carried out exclusively in the more natural $(r, \theta, t)$ coordinates.

\section{Appendix B. Convergence check for mode truncation}

Here, we check the convergence for the truncation of the SVD modes. The number of SVD modes generated by the SVD process is equal to the number of time steps. In the present study, we address the data from $t=3000$ to $t=4000$ with $\Delta t=1$, where $\Delta t$ is the time step, so that one thousand SVD modes are produced. This is clearly excessive in relation to the objective of capturing low-dimensional dynamics, which provides the rationale for our use of SVD: truncation is therefore necessary. Importantly, the calculation of spatial derivatives of the SVD-decomposed data is central to our analysis of the nonlinear physics. It follows that mode truncation must be robustly convergent in two senses: in reconstructing the original data; and in relation to spatial derivative variables. We note that in order to capture physical effects that involve higher order spatial derivatives, the retention of finer structure is necessary. Conversely, for spatially 
integrated quantities, convergence under truncation is faster than for spatial structures and their derivatives.

Let us denote the reconstructed data under truncation at the $M$ th SVD mode as

$$
\phi^{(M)}=\sum_{j=1}^{M} s_{j} \Psi_{j} h_{j} .
$$

We calculate the turbulence stress $\Pi_{r, \theta}^{(M)}$ (which requires a first order spatial derivative) and the vorticity $\Omega^{(M)}$ (involving the second derivative), using the reconstructed data $\phi^{(M)}$,

$$
\begin{aligned}
& \Pi_{r, \theta}^{(M)}=-\partial_{r} \phi^{(M)} \frac{1}{r} \partial_{\theta} \phi^{(M)}, \\
& \Omega^{(M)}=\nabla_{\perp}^{2} \phi^{(M)} .
\end{aligned}
$$

We then compare these with the corresponding quantities calculated from the original data $\phi$. Their deviations are evaluated from

$$
\epsilon_{X}(M)=\sqrt{\frac{\left\langle\int\left|X^{(M)}-X\right|^{2} d x^{3}\right\rangle_{t}}{\left\langle\int|X|^{2} d x^{3}\right\rangle_{t}}} .
$$

Here $X$ corresponds to $\phi, \Pi_{r, \theta}, \Omega$ calculated from the original data, whereas $X^{(M)}$ is obtained under SVD mode truncation at mode $M$, as in Eqs. (B.1) to (B.3), and $\langle\cdots\rangle_{t}$ denotes the time average. As shown in Fig. B1(a), the variables with the higher order derivatives converge more slowly.

Next, we check the convergence of the variables which involve spatial integration: the kinetic energy of the intermittent structure (mode D) and the energy transfer function between the background flow (mode A) and mode D. The spatio-temporal data of mode $\mathrm{D}$ under the mode truncation is introduced as

$$
\phi_{D}^{(M)}=\sum_{j=5}^{M} s_{j} \Psi_{j} h_{j} .
$$

The kinetic energy of mode D under truncation is denoted by $K_{D}^{(M)}$, and we denote the energy transfer to the background flow due to the self-nonlinearity of mode $\mathrm{D}$, under truncation, by $J^{(M)}(A \mid D, D)$. These are given by

$$
\begin{aligned}
& K_{D}^{(M)}=\frac{1}{2} \int\left|\nabla_{\perp} \phi_{D}^{(M)}\right|^{2} d^{3} x, \\
& J^{(M)}(A \mid D, D)=\int \phi_{A}\left[\phi_{D}^{(M)}, \nabla_{\perp}^{2} \phi_{D}^{(M)}\right] d^{3} x .
\end{aligned}
$$

The deviation of these quantities from those calculated from the original data is defined as

$$
\delta_{Y}^{(D)}(M)=\sqrt{\frac{\left\langle\left|Y^{(M)}-Y\right|^{2}\right\rangle_{t}}{\left\langle|Y|^{2}\right\rangle_{t}}},
$$

where $Y$ corresponds to $K_{D}$ and $J(A \mid D, D)$. The deviation $\delta_{Y}^{(D)}$ is plotted in Fig. $\mathrm{B} 1(\mathrm{~b})$. This shows that, empirically, their convergence is faster than for the spatial variables with similar trends, even though the energy transfer function has a higher 

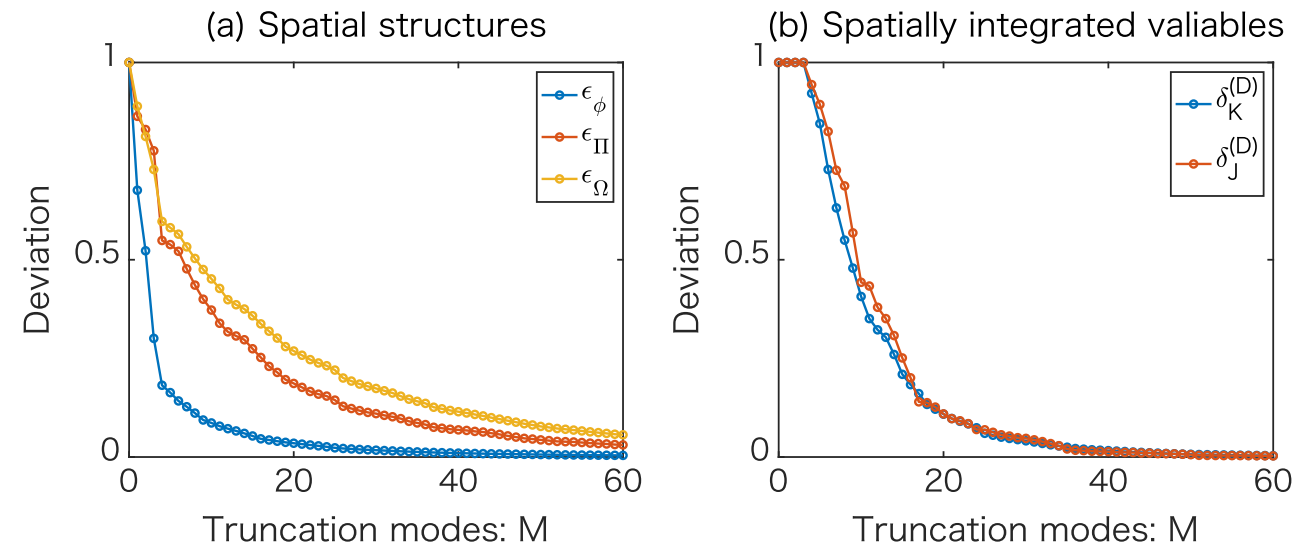

Figure B1. Difference between the input data and the reconstructed data, plotted against the SVD mode number $\mathrm{M}$ at which the SVD decomposition is truncated: (a) for spatila structures; (b) for spatially integrated variables.

order derivative (third order). In the present study, we therefore truncate the SVD mode decomposition at $M=40$ in order to retain $1 \%$ accuracy for the spatially integrated variables.

\section{Appendix C. Derivation of full set of energy equations}

The energy equations for the SVD modes are systematically derived in this appendix. The basic equations used in this simulation are given as [28]

$$
\begin{gathered}
\partial_{t} N=-[\phi, N]-D \nabla_{\|}^{2}(\phi-N)+\mu_{N} \nabla_{\perp}^{2} N+S_{N}, \\
\partial_{t} \nabla_{\perp}^{2} \phi=-\left[\phi, \nabla_{\perp}^{2} \phi\right]-D \nabla_{\|}^{2}(\phi-N)-\nu_{i n} \nabla_{\perp}^{2} \phi-\nu_{i n} \nabla N \cdot \nabla_{\perp} \phi \\
\quad-\nabla N \cdot d_{t} \nabla_{\perp} \phi+\mu_{U} \nabla_{\perp}^{4} \phi+S_{U},
\end{gathered}
$$

where $D$ is the electron parallel diffusion, $\mu_{N}$ and $\mu_{U}$ are the diffusion coefficients for particle and vorticity, and $\nu_{i n}$ is the ion-neutral collision frequency. The particle and vorticity sources are given by $S_{N}$ and $S_{U}$. Here, we decompose the fields, $N$ and $\phi$, with the SVD modes as

$$
\begin{aligned}
& N=\sum_{\zeta} N_{\zeta}, \\
& \phi=\sum_{\zeta} \phi_{\zeta},
\end{aligned}
$$

where $N_{\zeta}$ and $\phi_{z e t a}$ are the SVD modes for density and potential. It is noted that each SVD mode satisfies the orthogonality condition as

$$
\int \phi_{\zeta} \phi_{\zeta^{\prime}} d x^{3}=\int N_{\zeta} N_{\zeta^{\prime}} d x^{3}=\delta_{\zeta, \zeta^{\prime}},
$$

where $\zeta^{\prime}$ is the Kronecker delta. Multiplying $N_{\zeta}$ and $\phi_{\zeta}$ to density and vorticity equation, respectively, and performing the spatial integrals, the following energy equations are 
obtained. In the derivation, the orthogonality condition is used.

$$
\begin{aligned}
& \partial_{t} \int \frac{1}{2} N_{\zeta}^{2} d^{3} x=-\sum_{\alpha, \beta} \int N_{\zeta}\left[\phi_{\alpha}, N_{\beta}\right] d^{3} x+\int N_{\zeta} S_{N} d^{3} x \\
& \quad-D \sum_{\zeta^{\prime}} \int N_{\zeta} \nabla_{\|}^{2}\left(\phi_{\zeta^{\prime}}-N_{\zeta^{\prime}}\right)-\mu_{N} \int\left|\nabla_{\perp} N_{\zeta}\right|^{2} d^{3} x-\sum_{\zeta \neq \zeta^{\prime}} \int N_{\zeta} \partial_{t} N_{\zeta^{\prime}}(C .6) \\
& \partial_{t} \int \frac{1}{2}\left|\nabla_{\perp} \phi_{\zeta}\right|^{2} d^{3} x=\sum_{\alpha, \beta} \int \phi_{\zeta}\left[\phi_{\alpha}, \nabla_{\perp} \phi_{\beta}\right]-\nu_{i n} \int\left|\nabla_{\perp} \phi_{\zeta}\right|^{2} d^{3} x \\
& \quad-\int \phi_{\zeta} S_{U} d^{3} x+F_{\zeta}
\end{aligned}
$$

where $F_{\zeta}$ is given as

$$
\begin{aligned}
F_{\zeta}= & \sum_{\alpha, \beta} \int \phi_{\zeta}\left[\nu_{i n} \nabla N_{\alpha} \cdot \nabla_{\perp} \phi_{\beta}+\nabla N_{\alpha} \cdot d_{t} \nabla_{\perp} \phi_{\beta}\right] d^{3} x \\
& +\sum_{\zeta^{\prime}} \int \phi_{\zeta}\left[D \nabla_{\|}^{2}\left(\phi_{\zeta^{\prime}}-N_{\zeta^{\prime}}\right)-\mu_{U} \nabla_{\perp}^{4} \phi_{\zeta^{\prime}}\right] d^{3} x \\
& +\sum_{\zeta \neq \zeta^{\prime}} \int \nabla_{\perp} \phi_{\zeta} \cdot \partial_{t} \nabla_{\perp} \phi_{\zeta^{\prime}} d^{3} x .
\end{aligned}
$$

These are the full set of energy equation. In this study, only the potential is decomposed with the SVD, and we focus on the convective nonlinearity. The full analysis using the multi-field SVD analysis for the density and potential could be future work. 


\section{Reference}

[1] Sanae-I Itoh. Structure formation in turbulent plasmas. Plasma and Fusion Research, 4:038-038, 2009.

[2] Patrick H Diamond, SI Itoh, K Itoh, and TS Hahm. Zonal flows in plasma-a review. Plasma Physics and Controlled Fusion, 47(5):R35, 2005.

[3] ÖD Gürcan, PH Diamond, TS Hahm, and R Singh. Intrinsic rotation and electric field shear. Physics of Plasmas, 14(4):042306, 2007.

[4] X Litaudon. Internal transport barriers: critical physics issues? Plasma Physics and Controlled Fusion, 48(5A):A1, 2006.

[5] Fritz Wagner. A quarter-century of h-mode studies. Plasma Physics and Controlled Fusion, 49(12B):B1, 2007.

[6] Hamed Biglari, PH Diamond, and PW Terry. Influence of sheared poloidal rotation on edge turbulence. Physics of Fluids B: Plasma Physics, 2(1):1-4, 1990.

[7] H Imamura. Introduction to nonlinear analysis for engineerings, suurikougakusha-sha co.,ltd. Applied Mathematics, 16(2):185-186, 2006.

[8] K Miki, PH Diamond, S-H Hahn, WW Xiao, Özgür D Gürcan, and GR Tynan. Physics of stimulated l $\rightarrow$ h transitions. Physical Review Letters, 110(19):195002, 2013.

[9] Makoto Sasaki, K Itoh, S-I Itoh, and Naohiro Kasuya. Zonal flows induced by symmetry breaking with existence of geodesic acoustic modes. Nuclear Fusion, 52(2):023009, 2012.

[10] T Dudok de Wit, A-L Pecquet, J-C Vallet, and R Lima. The biorthogonal decomposition as a tool for investigating fluctuations in plasmas. Physics of Plasmas, 1(10):3288-3300, 1994.

[11] DR Hatch, MJ Pueschel, F Jenko, WM Nevins, PW Terry, and H Doerk. Magnetic stochasticity and transport due to nonlinearly excited subdominant microtearing modes. Physics of Plasmas, 20(1):012307, 2013.

[12] B Ph Van Milligen, E Sánchez, A Alonso, MA Pedrosa, C Hidalgo, A Martín De Aguilera, and A López Fraguas. The use of the biorthogonal decomposition for the identification of zonal flows at tj-ii. Plasma Physics and Controlled Fusion, 57(2):025005, 2014.

[13] S Ohdachi, K Toi, G Fuchs, TEXTOR team, LHD Experimental Group, et al. Magnetic islands observed by a fast-framing tangentially viewing soft x-ray camera on lhd and textor. Plasma Science and Technology, 8(1):45, 2006.

[14] C Marchetto, E Alessi, L Boncagni, C Galperti, S Nowak, and C Sozzi. Application of svd algorithm to a set of real time mirnov coil signals in ftu tokamak. In EPS Conf. on Plasma Physics, pages 2-64, 2012.

[15] S Gerksic et al. Svd-based fast online mpc for iter plasma current and shape control.

[16] MA Athanasiu and GP Pavlos. Svd analysis of the magnetospheric ae index time series and comparison with low-dimensional chaotic dynamics. Nonlinear Processes in Geophysics, 8:95125, 2001.

[17] M Sasaki, Y Kawachi, RO Dendy, H Arakawa, N Kasuya, F Kin, K Yamasaki, and S Inagaki. Using dynamical mode decomposition to extract the limit cycle dynamics of modulated turbulence in a plasma simulation. Plasma Physics and Controlled Fusion, 61(11):112001, 2019.

[18] Akira Kusaba, Tetsuji Kuboyama, and Shigeru Inagaki. Sparsity-promoting dynamic mode decomposition of plasma turbulence. Plasma and Fusion Research, 15:1301001-1301001, 2020.

[19] Alan A Kaptanoglu, Kyle D Morgan, Chris J Hansen, and Steven L Brunton. Characterizing magnetized plasmas with dynamic mode decomposition. Physics of Plasmas, 27(3):032108, 2020.

[20] Diego del Castillo-Negrete, Steven P Hirshman, Donald A Spong, and Eduardo F D ' Azevedo. Compression of magnetohydrodynamic simulation data using singular value decomposition. Journal of Computational Physics, 222(1):265-286, 2007.

[21] DR Hatch, F Jenko, A Bañón Navarro, V Bratanov, PW Terry, and MJ Pueschel. Linear signatures in nonlinear gyrokinetics: interpreting turbulence with pseudospectra. New Journal of Physics, 
18(7):075018, 2016.

[22] Yoshiki Kuramoto. Chemical oscillations, waves, and turbulence. Courier Corporation, 2003.

[23] A Fujisawa, H Iguchi, H Idei, S Kubo, K Matsuoka, S Okamura, K Tanaka, T Minami, S Ohdachi, S Morita, et al. Discovery of electric pulsation in a toroidal helical plasma. Physical Review Letters, 81(11):2256, 1998.

[24] T Kobayashi, K Itoh, T Ido, K Kamiya, S-I Itoh, Y Miura, Yoshihiko Nagashima, A Fujisawa, S Inagaki, K Ida, et al. Spatiotemporal structures of edge limit-cycle oscillation before l-to-h transition in the jft-2m tokamak. Physical Review Letters, 111(3):035002, 2013.

[25] L Schmitz, L Zeng, TL Rhodes, JC Hillesheim, EJ Doyle, RJ Groebner, WA Peebles, KH Burrell, and G Wang. Role of zonal flow predator-prey oscillations in triggering the transition to h-mode confinement. Physical Review Letters, 108(15):155002, 2012.

[26] G Birkenmeier, M Cavedon, GD Conway, P Manz, U Stroth, R Fischer, G Fuchert, T Happel, FM Laggner, M Maraschek, et al. Magnetic structure and frequency scaling of limit-cycle oscillations close to l-to h-mode transitions. Nuclear Fusion, 56(8):086009, 2016.

[27] Sanae-I Itoh, Kimitaka Itoh, Atsushi Fukuyama, and Yukitoshi Miura. Edge localized mode activity as a limit cycle in tokamak plasmas. Physical Review Letters, 67(18):2485, 1991.

[28] Makoto Sasaki, Yann Camenen, A Escarguel, S Inagaki, Naohiro Kasuya, K Itoh, and T Kobayashi. Formation of spiral structures of turbulence driven by a strong rotation in magnetically cylindrical plasmas. Physics of Plasmas, 26(4):042305, 2019.

[29] Akira Hasegawa and Masahiro Wakatani. Self-organization of electrostatic turbulence in a cylindrical plasma. Physical Review Letters, 59(14):1581, 1987.

[30] W Horton, T Tajima, and T Kamimura. Kelvin-helmholtz instability and vortices in magnetized plasma. The Physics of Fluids, 30(11):3485-3495, 1987.

[31] Makoto Sasaki, Naohiro Kasuya, K Itoh, S Toda, Takuma Yamada, Yusuke Kosuga, Yoshihiko Nagashima, T Kobayashi, H Arakawa, K Yamasaki, et al. Topological bifurcation of helical flows in magnetized plasmas with density gradient and parallel flow shear. Physics of Plasmas, 24(11):112103, 2017.

[32] K Itoh, S-I Itoh, Yusuke Kosuga, M Lesur, and T Ido. Onset condition of the subcritical geodesic acoustic mode instability in the presence of energetic-particle-driven geodesic acoustic mode. Plasma Physics Reports, 42(5):418-423, 2016.

[33] RO Dendy, SC Chapman, and S Inagaki. Modelling the measured local time evolution of strongly nonlinear heat pulses in the large helical device. Plasma Physics and Controlled Fusion, 55(11):115009, 2013.

[34] H Zhu, RO Dendy, SC Chapman, and S Inagaki. A quantitative model for heat pulse propagation across large helical device plasmas. Physics of Plasmas, 22(6):062308, 2015.

[35] P Manz, M Ramisch, and U Stroth. Physical mechanism behind zonal-flow generation in drift-wave turbulence. Physical Review Letters, 103(16):165004, 2009.

[36] Yoshihiko Nagashima, Sanae-I Itoh, Shunjiro Shinohara, Masayuki Fukao, Akihide Fujisawa, Kenichiro Terasaka, Yoshinobu Kawai, George R Tynan, Patrick H Diamond, Masatoshi Yagi, et al. Observation of the parametric-modulational instability between the drift-wave fluctuation and azimuthally symmetric sheared radial electric field oscillation in a cylindrical laboratory plasma. Physics of Plasmas, 16(2):020706, 2009.

[37] Eun-jin Kim and PH Diamond. Zonal flows and transient dynamics of the $\mathrm{l}-\mathrm{h}$ transition. Physical Review Letters, 90(18):185006, 2003.

[38] T Kobayashi, S Inagaki, M Sasaki, Y Kosuga, H Arakawa, T Yamada, Y Nagashima, Y Miwa, N Kasuya, A Fujisawa, et al. Azimuthal inhomogeneity of turbulence structure and its impact on intermittent particle transport in linear magnetized plasmas. Physics of Plasmas, 22(11):112301, 2015.

[39] H Arakawa, S Inagaki, M Sasaki, Y Kosuga, T Kobayashi, N Kasuya, Y Nagashima, T Yamada, M Lesur, A Fujisawa, et al. Eddy, drift wave and zonal flow dynamics in a linear magnetized plasma. Scientific Reports, 6(1):33371, 2016. 
[40] H Arakawa, M Sasaki, S Inagaki, Y Kosuga, T Kobayashi, N Kasuya, T Yamada, Y Nagashima, F Kin, A Fujisawa, et al. Roles of solitary eddy and splash in drift wave-zonal flow system in a linear magnetized plasma. Physics of Plasmas, 26(5):052305, 2019.

[41] JRK Kumar Dabbakuti, Venkata Ratnam Devanaboyina, and S Ramesh Kanchumarthi. Analysis of local ionospheric variability based on svd and mds at low-latitude gnss stations. Earth, Planets and Space, 68(1):1-11, 2016.

[42] SI Itoh and K Itoh. A mini-max principle for drift waves and mesoscale fluctuations. Plasma Physics and Controlled Fusion, 53(1):015008, 2010.

[43] K Itoh, K Hallatschek, S-I Itoh, PH Diamond, and S Toda. Coherent structure of zonal flow and onset of turbulent transport. Physics of Plasmas, 12(6):062303, 2005.

[44] JS Kim, DH Edgell, JM Greene, EJ Strait, and MS Chance. MHD mode identification of tokamak plasmas from Mirnov signals. Plasma Physics and Controlled Fusion, 41(11):1399, 1999.

[45] GS Xu, BN Wan, W Zhang, QW Yang, L Wang, and YZ Wen. Multiscale coherent structures in tokamak plasma turbulence. Physics of Plasmas, 13(10):102509, 2006.

[46] DR Hatch, F Jenko, A Bañón Navarro, V Bratanov, PW Terry, and MJ Pueschel. Linear signatures in nonlinear gyrokinetics: interpreting turbulence with pseudospectra. New Journal of Physics, 18(7):075018, 2016. 